\title{
3D imaging of the fetal face - Recommendations from the International 3D Focus Group
}

Key words

- 3D ultrasound

- fetus

- face

\section{Introduction}

Three-dimensional and four-dimensional ultrasound ( $3 \mathrm{D} / 4 \mathrm{D}$ US) technology can be easily applied to routine prenatal diagnosis and can provide images of the fetal face that cannot be achieved with two-dimensional ultrasound (2 D US) [1 -7]. The most recent $3 \mathrm{D} / 4 \mathrm{D}$ US technology facilitates the $3 \mathrm{D} / 4 \mathrm{D}$ US examinations even for the less trained sonographer and gives the examiner the opportunity to identify the normal and abnormal fetal face in the most appropriate imaging mode [8].

The purpose of publishing these recommendations was to demonstrate the different possibilities of assessing the normal and abnormal fetal face with $3 \mathrm{D} / 4 \mathrm{D}$ ultrasound and to give the operator an overview of the benefit resulting from the application of that technology.

\section{Display modes}

In contrast to conventional $2 \mathrm{D}$ US, which provides only one imaging mode to demonstrate the fetus, 3D/4D US technology offers the ability to review the fetal face in different display modes $[7,8]$ : 1 . three-orthogonal-plane $\left(=\operatorname{triplanar}^{-1}\right.$ or multiplanar $^{1}$ ) display, 2 . parallel-plane (= tomographic $^{1}$ or multislice ${ }^{1}$ ) display, 3. $3 \mathrm{D}$ surface display and 4. $3 \mathrm{D}$ transparent display (maximum, $\mathrm{X}$ ray and minimum mode). In addition some ultrasound systems allow also an 'any plane slicing' and a mixing of two different modes (eg. mode 1 $70 \%$ and mode $230 \%$ ). Furthermore 4D US allows volume visualization of $3 \mathrm{D}$ images of the fetus in real-time.

\section{Three-orthogonal-plane display}

In this display $[7,8]$ all three mutually perpendicular planes are displayed simultaneously on the monitor immediately after volume acquisition in $3 \mathrm{D}$ or in $4 \mathrm{D}$ (real time) US scanning. In this pri- mary 3-orthogonal-plane image ( $\bullet$ Fig. 1) [9], the object is displayed so that the image at the top left always corresponds to that visualized in the monitor during $2 \mathrm{D}$ US scanning. This permits to draw a valid conclusion on the position of the embryo/fetus at the time of volume scanning. Following display of all 3 perpendicular planes the object can be rotated in all 3 dimensions. However, this can lead to a loss in spatial orientation. To avoid such a confusion the ISUOG 3 D Focus Group [9] has recommended to rotate the volume in such a way that the embryo/fetus may at all times be visualized in an upright sagittal position in the image at the top right (secondary 3-orthogonal-plane image) ( $\bullet$ Fig. 2 ). This ensures that the object of interest is visualized automatically in an anatomically correct position in the coronal and transverse plane, regardless of the possible vertex or breech presentation.

With the three-orthogonal-plane display all conventional scan planes can be viewed, as well as planes that are unattainable with traditional 2D US. The simultaneous display of all three perpendicular sectional planes provides an ideal basis for a detailed survey of the fetus and allows the demonstration of a specific $2 \mathrm{D}$ plane to be precisely controlled. To optimize the position of the face, the cursor dot should be placed between the orbits in the coronal plane and the face rotated in all planes until it is symmetrical ( $\bullet$ Fig. 2).

\section{Parallel-plane (= tomographic) display} With the parallel-plane display $[7,8]$ the region of interest can be displayed on the monitor in several parallel slices similarly to CT or MRI ( $\bullet$ Fig. 35). This display enables a detailed tomographic survey of the fetal face in sagittal, coronal and transverse slices at different distances. The sagit-

\footnotetext{
1 These terms are widely used simultaneously. However, from the logical point of view, they are not as precise as the main term.
} 
tal planes are helpful to demonstrate the profile and the orbits, the coronal planes to assess the orbits and the palate and the transverse planes to evaluate the orbits, palate, maxilla and mandible.

\section{3. $3 \mathrm{D}$ surface display}

This display mode $[7,8]$ provides three-dimensional surface images of the fetal face ( $\bullet$ Fig. 6,7 ). There are different rendering (calculation) algorithms to depict the fetal face: surface mode, soft surface mode, light mode, and soft light mode ( $\bullet$ Fig. 8). Two of these modes can be mixed in different percentages.

The prerequisites for high-quality $3 \mathrm{D}$ US images of the fetal surface are the presence of a sufficient amniotic fluid pocket in front of the structure being imaged and the absence of overlying or adjacent structures [2]. However, in some cases the face is hidden by overlying or adjacent structures. In order to obtain a high quality surface image those structures have to be removed with the electronic scalpel [10]. This tool allows the examiner to remove

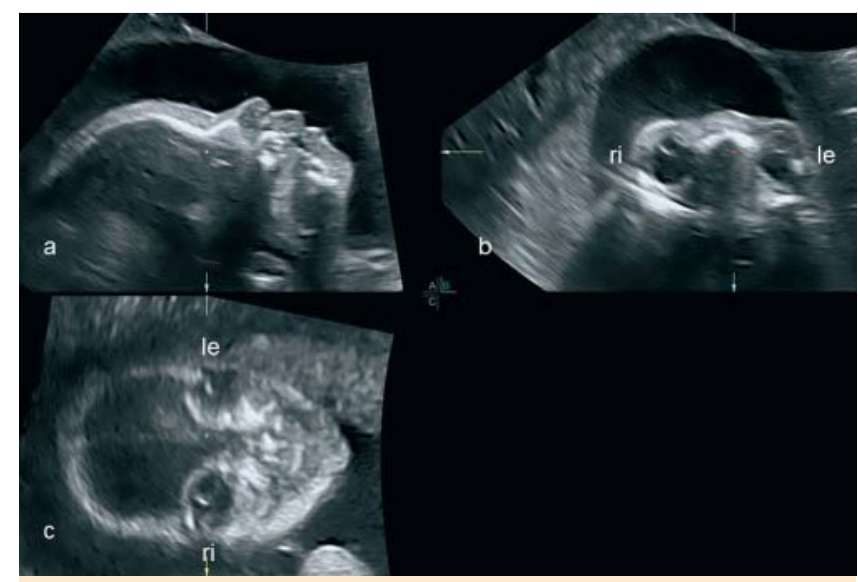

Fig. 1 Three-orthogonal-plane display of a fetus in breech presentation at 25 weeks of gestation. Primary 3-orthogonal-plane image after acquisition. Plane $\mathbf{a}=$ sagittal plane. Plane $\mathbf{b}=$ transverse plane (bird's eye view). Plane $\mathbf{c}=$ coronal plane $(=$ frontal view). Plane $\mathbf{a}$ demonstrates the $2 \mathrm{D}$ US image during acquisistion. le $=$ left, $\mathrm{ri}=$ right. most of the overlaying structures in any of the slice images, or in the $3 \mathrm{D}$ image itself. However, when overlaying structures (eg. limb bones) create acoustic shadows, the electronic scalpel cannot overcome those artifacts. The best surface images can be achieved when the fetal face is looking towards the probe, when there is enough amniotic fluid in front of the face and when there are no overlaying structures causing shadows.

\section{Transparent display}

In terms of the different transparent modes (maximum mode, $\mathrm{X}$ ray mode), the maximum mode (= maximum intensity display) $[7,8,11-13]$ can be used to visualize hyperechogenic structures. This mode provides a complete survey of the fetal skull and facial bones ( $\nabla$ Fig. 9). To get high quality pictures this mode can be mixed with surface mode.

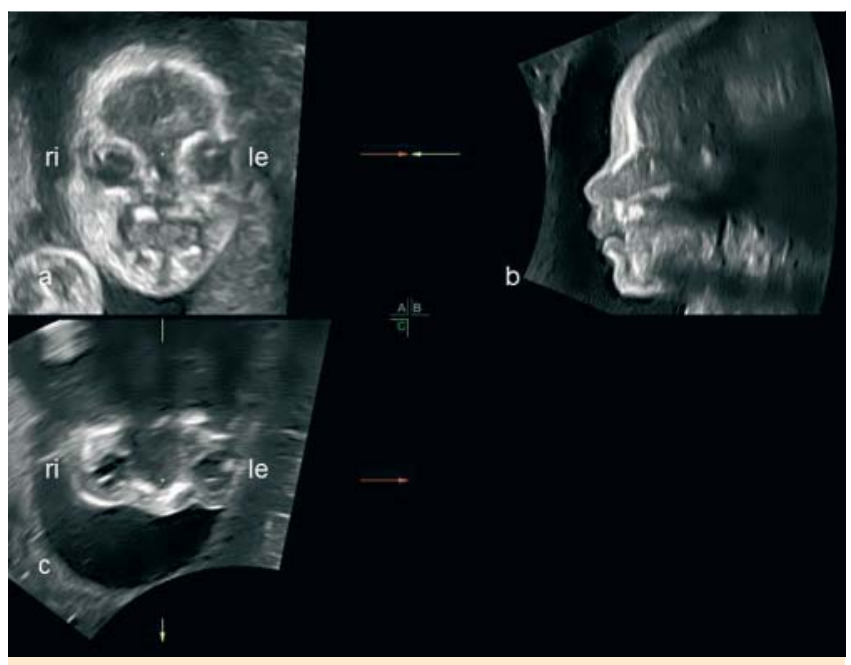

Fig. 2 Secondary 3-orthogonal-plane image after rotation the fetus of Fig. 1 into an upright position and horizontal rotation of $90^{\circ}$. Plane $\mathbf{a}=\mathrm{co-}$ ronal plane at orbit level. Plane $\mathbf{b}=$ median plane. Plane $\mathbf{c}=$ transverse plane at orbit level. This demonstration shows the fetal face always in an anatomically correct position: le = left, ri = right.

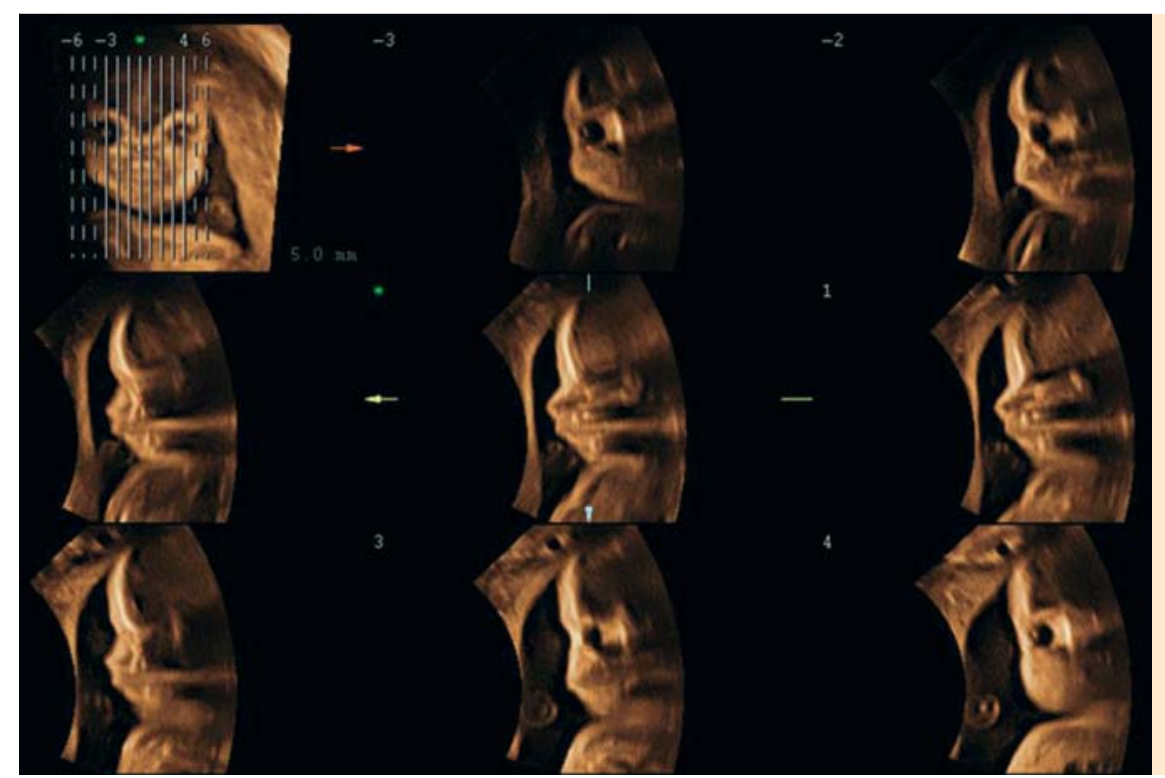

Fig. 3 Parallel-plane (= tomographic) display showing several slices of the fetal face at distances of $5.0 \mathrm{~mm}$ (sagittal views). 23 weeks of gestation. 


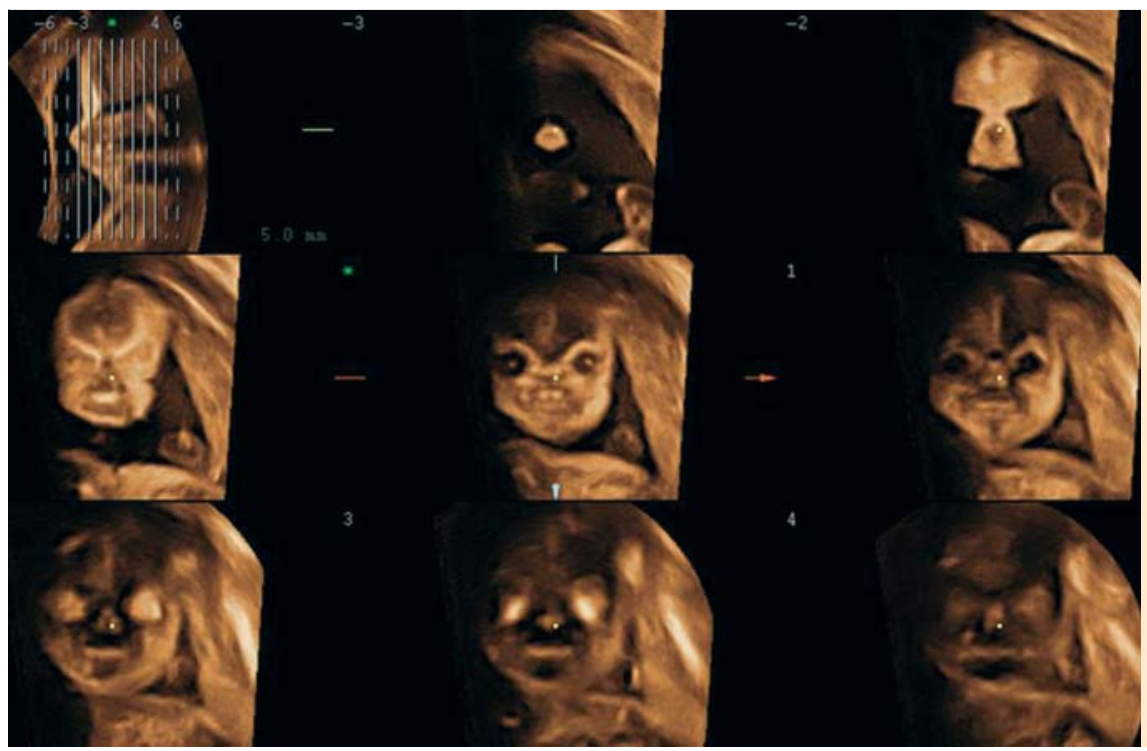

Fig. 4 Parallel-plane (= tomographic) display showing several slices of the fetal face at distances of $5.0 \mathrm{~mm}$ (coronal views). 23 weeks of gestation.

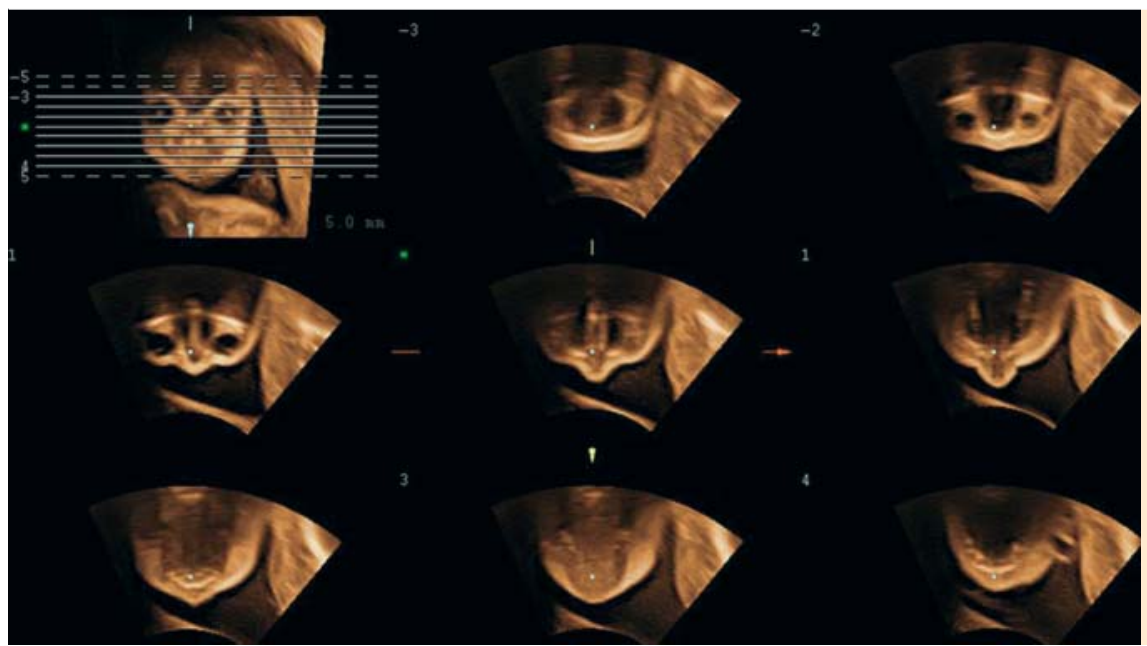

Fig. 5 Parallel-plane (= tomographic) display showing several slices of the fetal face at distances of $5.0 \mathrm{~mm}$ (transverse views). 23 weeks of gestation.

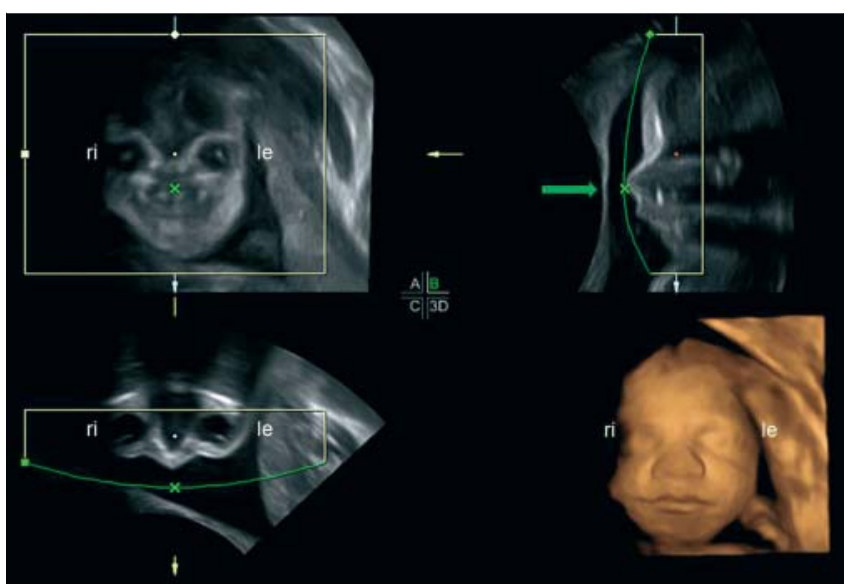

Fig. 6 Three-orthogonal-plane display and 3 D US surface rendered image of a fetus at 23 weeks of gestation after rotation the face into an upright position and horizontal rotation of $90^{\circ}$. Upper left: Coronal image of face. Upper right: Sagittal view. The volume box is positioned close to the surface of the face. The green arrow and the green line demonstrate the camera position. Lower left: Axial view of the orbits. Lower right: A surfacerendered image of the fetal face (frontal view). le $=$ left, ri $=$ right.
$4 \mathrm{D}$ ultrasound (= real time $3 \mathrm{D}$ US) $[7,8]$

$4 \mathrm{D}$ US technology allows real time visualization of the fetus in any of the modes described above. In particular, with fast acquisition of up to 35 volumes per second, both the surface and movements of the fetus can be demonstrated constantly on the screen. This enables the physician to study fetal facial movements such as yawning, sucking, swallowing, mimicking [9] and offers the parents the opportunity to observe fetal movements. From the diagnostic point of view 4D US is always helpful in the moving fetus, and $3 \mathrm{D}$ US in the fetus at rest.

\section{D US imaging of the fetal face in the first trimester (11 - 14 weeks of gestation)}

\section{Normal anatomy}

In the first trimester 3D US can be applied for the profile and for demonstration of normal ossification ( $\bullet$ Table 1 ). While the profile with nose, lips and chin can be shown with the three-orthogonalplane display and a 3D US surface rendered image as well ( 0 Fig. 10), the demonstration of the nasal bone $[14,15]$ and the frontomaxillary facial (= FMF) angle $[16,17]$ is performed using the 3-orthogonal-plane display ( $\boldsymbol{0}$ Fig. 11, 12). To demonstrate 


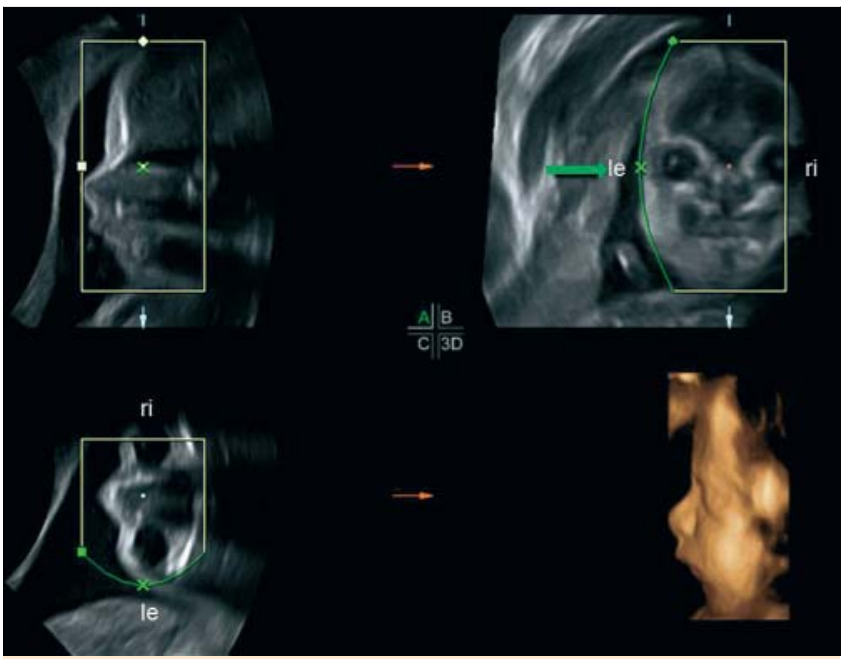

Fig. 7 Three-orthogonal-plane display and 3 D US surface rendered image of a fetus at 23 weeks of gestation after rotation the face into an upright position (upper left). Upper right: The volume box for rendering the region of interest is positioned close to the surface of the left side of the face (reverse view). The green arrow and the green line demonstrate the camera position. Lower left: View of the orbits from a bird's eye view. le = left, ri = right. Lower right: A surface-rendered image of a fetal face (side view) in profile.

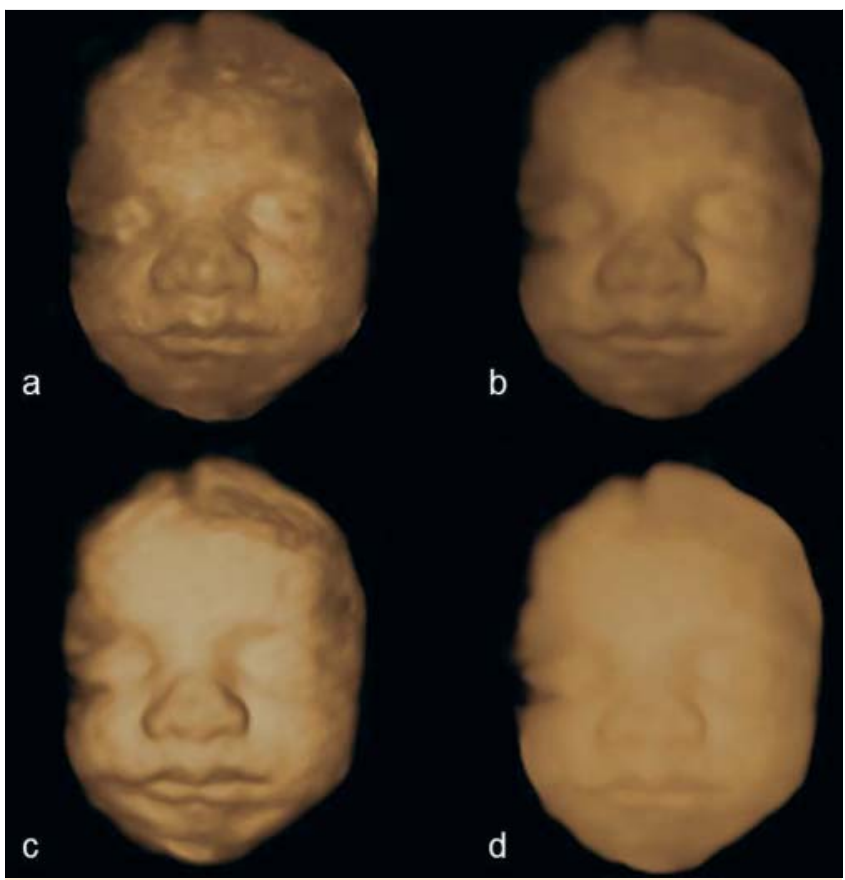

Fig. 8 Different surface displays of a fetus at 23 weeks of gestation. $\mathbf{a}=$ surface mode, $\mathbf{b}=$ soft surface mode, $\mathbf{c}=$ soft light mode, $\mathbf{d}=$ light mode.

\section{Early detection of malformations of the fetal face}

Absent nasal bone $[14,15,19]$ and increased FMF-angle $\left(>85^{\circ}\right)$ [17] in the first trimester are important screening signs for possible trisomy 21 . However, absent nasal bone is also found in $2.6 \%$ of euploid fetuses. Prevalence of absent nasal bone is affected not only by the fetal karyotype but also by maternal ethnicity, being higher in black than in white women [19]. Because some healthy fetuses show a delayed ossification of the facial bones it is recommended not to evaluate these two parameters before 12 weeks of gestation.

Using advanced ultrasound systems anomalies such as micrognathia, hypo- and hypertelorism, cleft lip and palate, can be diagnosed in some fetuses in the first trimester although there is much more experience in the second trimester (see below).

\section{D US imaging of the face, orbits, nasal bone, palate, maxilla and mandible in the second and third trime- sters}

\section{Normal anatomy}

In the second and third trimesters $3 \mathrm{D}$ US can be applied for the demonstration of the surface of the fetal face $[1,2,7]$ (๑ Fig. 6 8) and for the demonstration of a normal ossification of the facial bones as well [12] ( $\bullet$ Fig. 9). As in the first trimester the profile with the nose, lips and chin are shown in the 3-orthogonal plane display ( $\bullet$ Fig. 1, 2) and in the $3 \mathrm{D}$ surface rendered image as well. Orbits and lenses are easily compared in the coronal and transverse planes in the 3-orthogonal-plane display ( $\bullet$ Fig. 2). These planes can be also used to measure the orbit diameters and the inner and outer orbital distances. Measurement of the FMF-angle [16] can be performed as in the first trimester [17] ( $\bullet$ Fig. 12). Demonstration of the nasal bone is much easier, because both nasal bones are now fused and can be shown in a slightly parasagittal and in the median plane as well. The nasal bone can be shown 


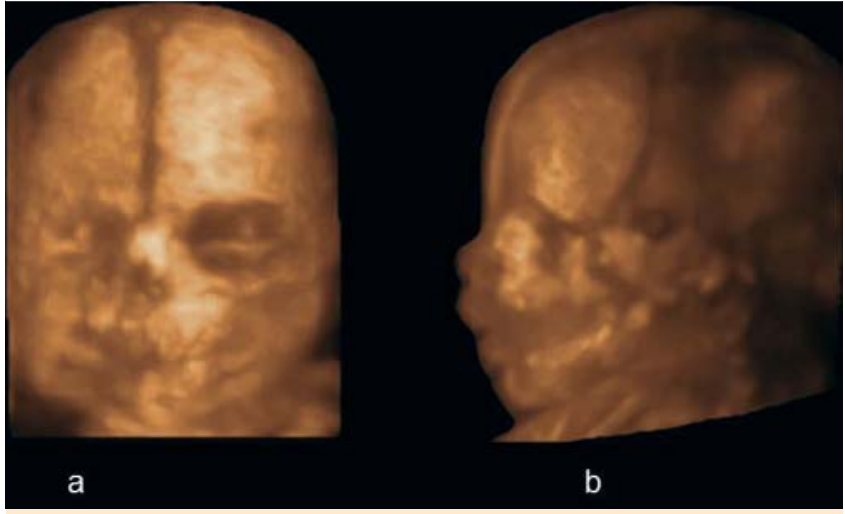

Fig.9 Transparent (= maximum intensity) display of a normal fetal head at 23 weeks of gestation. a The frontal view shows the echogenic ossified facial bones and the metopic suture. $\mathbf{b}$ The side view demonstrates the ossified skull, the facial bones and the left coronal suture.

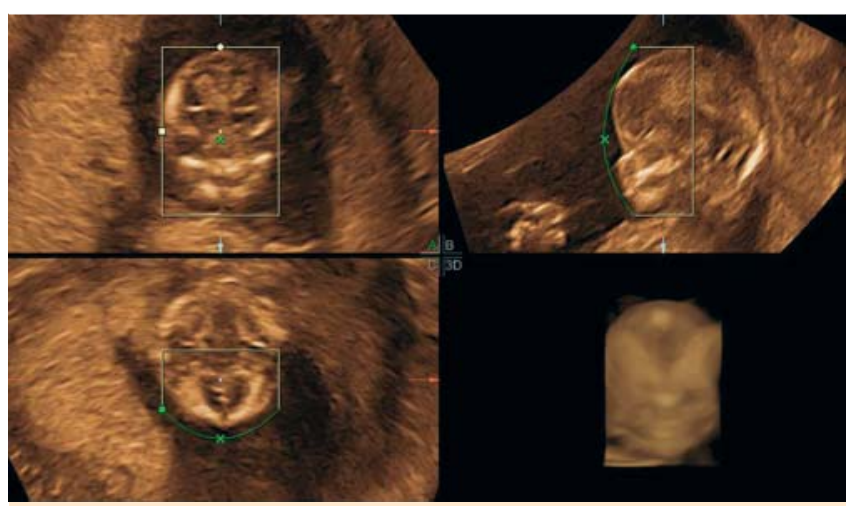

Fig. 10 Three-orthogonal-plane display and 3D surface image of a fetus at 13 weeks of gestation after rotation of the fetus into an upright position and horizontal rotation of $90^{\circ}$. Upper right and lower left: The volume box for surface rendering is positioned close to the surface of the face. The green line demonstrates the camera position. Lower right: A surface-rendered image of a fetal face (frontal view).

with the 3-orthogonal-plane display and also with the maximum intensity (transparent) display. Measurement of the nasal bones can be performed in the sagittal and coronal plane as well.

Demonstration of the palate is best performed by acquiring the volume so that the palate is not shadowed by other facial structures. This can be easily done in the sagittal plane while the head is tipped posteriorly. The palate can be shown from three different views using maximum intensity display: from a front view ( $\bullet$ Fig. 13), from a 'reverse face' view [20] ( $\bullet$ Fig. 14) or from a bottom view inside the mouth [21] ( $\bullet$ Fig. 15). For that purpose, a thin volume box is applied in the sagittal image of the 3 orthogonal planes as shown in 0 Fig.13-15. Further approaches to demonstrate the secondary palate are the 'flipped face view' technique $[22,24]$ and the angled insonation technique $[23,24]$ where the secondary palate is insonated at $45^{\circ}$ angle in the sagittal plane and $3 \mathrm{D}$ US is used to reconstruct axial and coronal planes. Another option is to demonstrate the secondary pa-

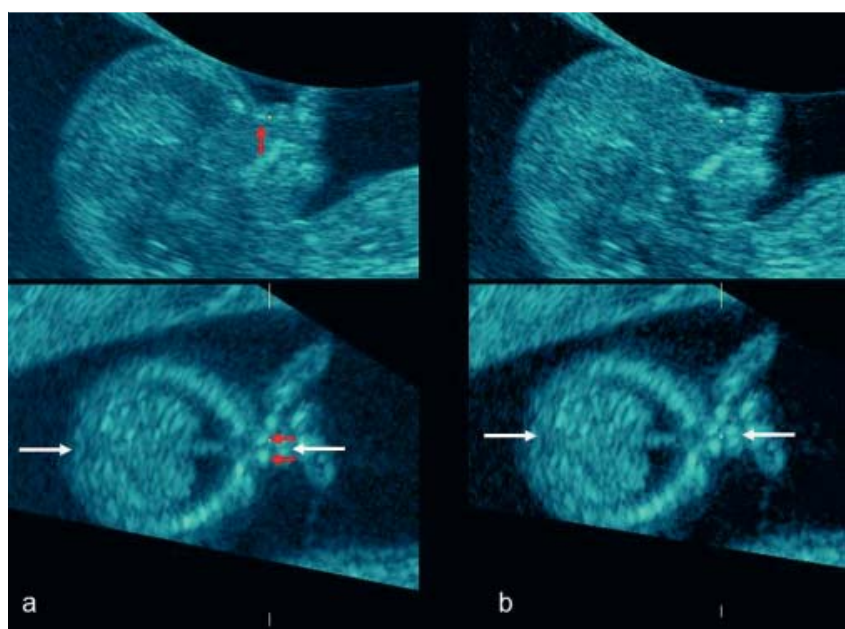

Fig. 11 Three-orthogonal-plane display of a fetus at $11+2$ weeks of gestation. a. Upper image: sagittal slightly paramedian scan plane with a rotation of the face of $45^{\circ}$ to ensure that the nasal bone (red arrow) is parallel to the upper image margin (white dot caudal of the left nasal bone). Lower image: The coronal scan plane reveals that the sagittal scan plane is running through the left nasal bone (upper red arrow, white dot). White arrows = median plane. b. Upper image: median plane. The nasal bone is not visible because the section plane is running exactly through the gap between the left and right nasal bone (see coronal plane in the lower image). Lower image: The white dot is confirming the true median section plane (white arrows).

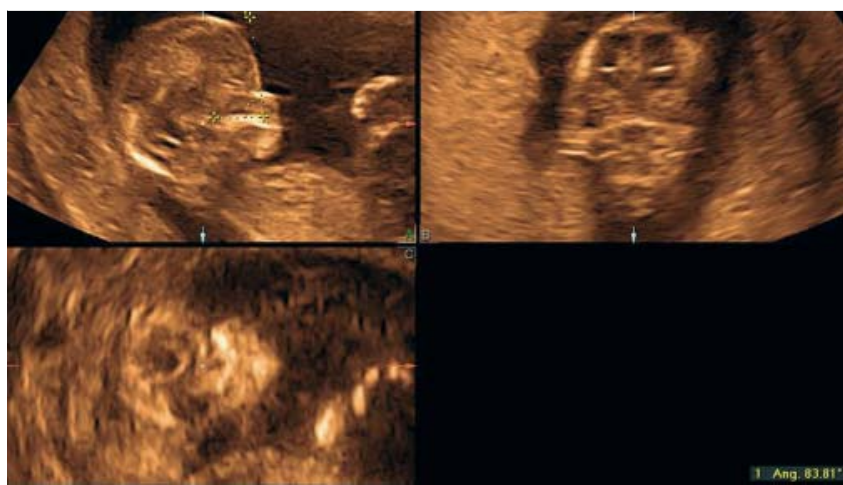

Fig. 12 Three-orthogonal-plane display of a fetus at 13 weeks of gestation. Upper left: sagittal scan with a rotation of about $60^{\circ}$ to ensure that the maxilla is parallel to the upper image margin. The frontomaxillar facial angle is measured between the upper surface of the maxilla and the external surface of the frontal bone; it measured $84^{\circ}$ in this case which is normal.

late in the $3 \mathrm{D}$ US surface rendered display by cutting the frontal part of the fetal face with the electronic scalpel $[8,10]$ ( $\bullet$ Fig. 16) or by placing the volume box inside the fetal face ( $\bullet$ Fig. 16).

In a detailed ultrasound examination for demonstration or exclusion of a fetal face mal formation the following four images are recommended for documentation: sagittal view with profile and nasal bone, coronal view with lips, coronal view with palate and transverse view with orbits ( $\bullet$ Table 2 ).

\section{Detection of fetal face anomalies}

In the assessment of fetal face anomalies, the operator performing the ultrasound examination can choose the most appropriate display mode [8]: 


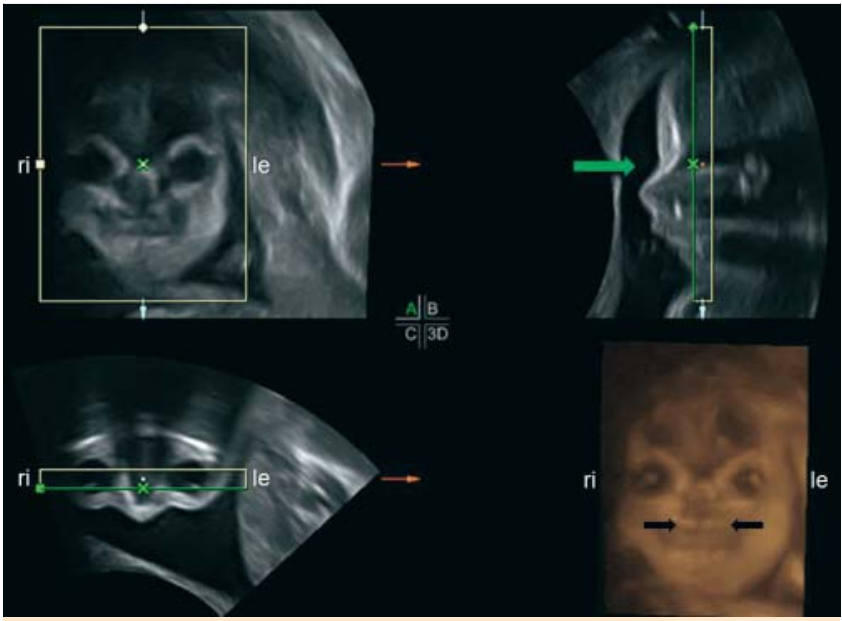

Fig. 13 Three-orthogonal-plane and transparent (= mix of maximum intensity and surface mode) display of a fetus at 23 weeks of gestation after rotation of the face into an upright position and horizontal rotation of $90^{\circ}$. Upper right: The volume box for rendering the inner surface is positioned inside the face to demonstrate the palate. The green arrow and the green line demonstrate the camera position. le = left, $r i=$ right. Lower right: Frontal view of the ossified palate (black arrows).

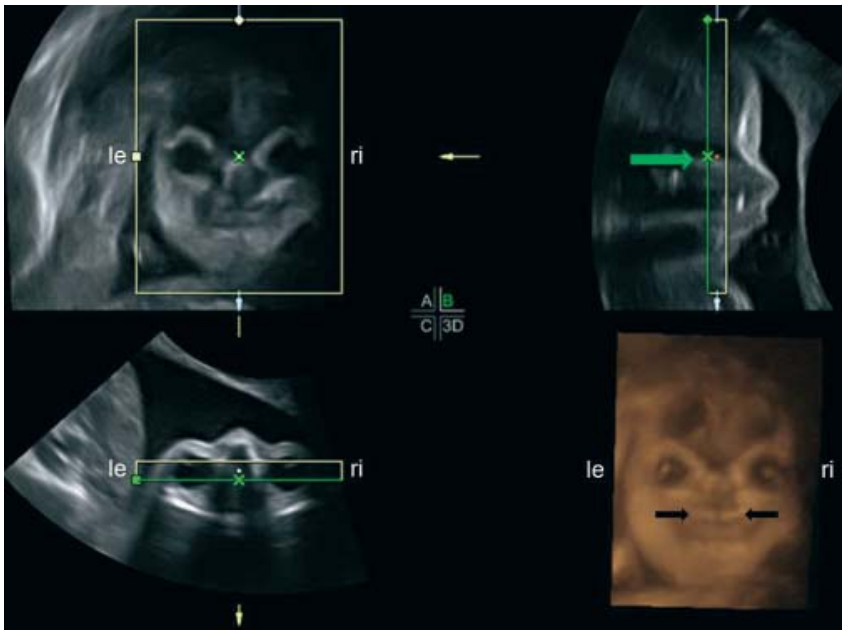

Fig. 14 Three-orthogonal-plane and transparent (= maximum intensity) display of a fetus at 23 weeks of gestation after rotation of the face into an upright position and rotation of $180^{\circ}$. Upper right: The ROI for rendering is positioned inside the face to demonstrate the palate from a reverse view. The green arrow and the green line demonstrate the camera position. Lower right: Reverse view of the ossified palate (black arrows). le = left, ri = right.

Simultaneous display of all three orthogonal sectional planes (3orthogonal-plane display) is particularly helpful in the verification of an exact mid-sagittal section (= median plane), which is required to reliably identify a flat facial profile or micrognathia $[2,6,7,8,18,25]$. In addition, mid-facial hypoplasia [26] or absence of the nasal bone [11] can be detected accurately and should serve as an indication for invasive testing for trisomy 21. The coronal and transverse planes allow the comparison of the orbit diameters and a conclusive demonstration of eye malformations. In order to avoid confusion regarding the accurate anatomical assignment of left/right and dorsal/ventral, it is helpful to use a standardized 3-orthogonal-plane display [9].

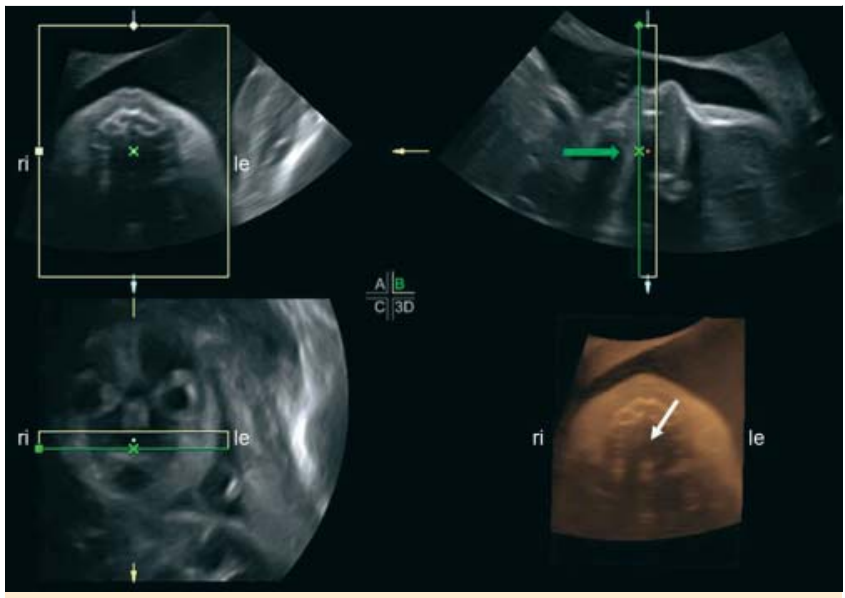

Fig. 15 Three-orthogonal-plane and surface display of the primary and secon-dary palate in a fetus at 23 weeks of gestation after rotation of the face into a horizontal position in Plane B (upper right). The volume box for rendering the $\mathrm{ROI}$ is positioned inside the face to demonstrate the palate from a bottom view. The green arrow and the green line demonstrate the camera position. Lower right: Bottom view of the ossified primary and hard palate (white arrow). le = left, ri $=$ right.

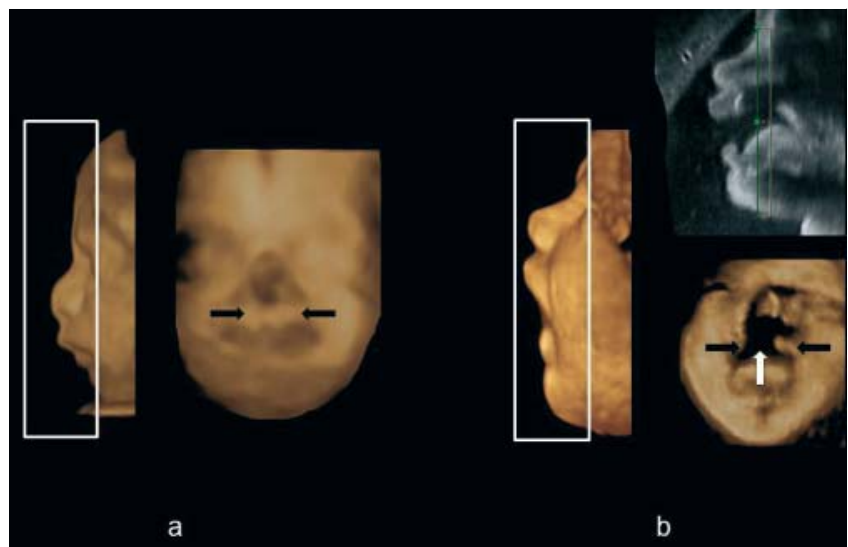

Fig. 16 a Surface-rendered side and frontal view of a normal fetus (23 weeks of gestation). The frontal view reveals the ossified palate after removing the frontal part of the fetal face with the electronic scalpel indicated by the white cutting box. $\mathbf{b}$ Surface-rendered side and frontal view of a fetus with isolated cleft palate right (white arrow) (25 weeks of gestation). Black arrows = ossified palate. The surface-rendered image is achieved either by cutting of the frontal part of the fetal face $\mathbf{a}$ or by positioning the volume box inside the face $\mathbf{b}$.

The parallel-plane (= tomographic) display can be useful in the demonstration of facial malformations as, e.g. hyper-/hypotelorism, orbital dysplasia, cataract, cleft lip and cleft palate. By selecting the number of parallel slices and the distance between the slices, the acquired region of interest (ROI) can be displayed on the monitor at different numbers of $2 \mathrm{D}$ US images, which provides a precise overview of the entire range of the acquired volume [7]. Surface rendered images accurately demonstrate all malformations on the surface of the fetal face either in a side or a frontal view: frontal bossing, flat profile, cyclopia with proboscis, cleft lip, micrognathia $[2,6]$. Rendering of multiple $3 \mathrm{D}$ US images (cine sequence) gives an excellent spatial depiction of the severity of a defect, as this can be viewed from different angles. For the 
Table 2 Assessment of the fetal face by 3 D US in the second and third trimesters.

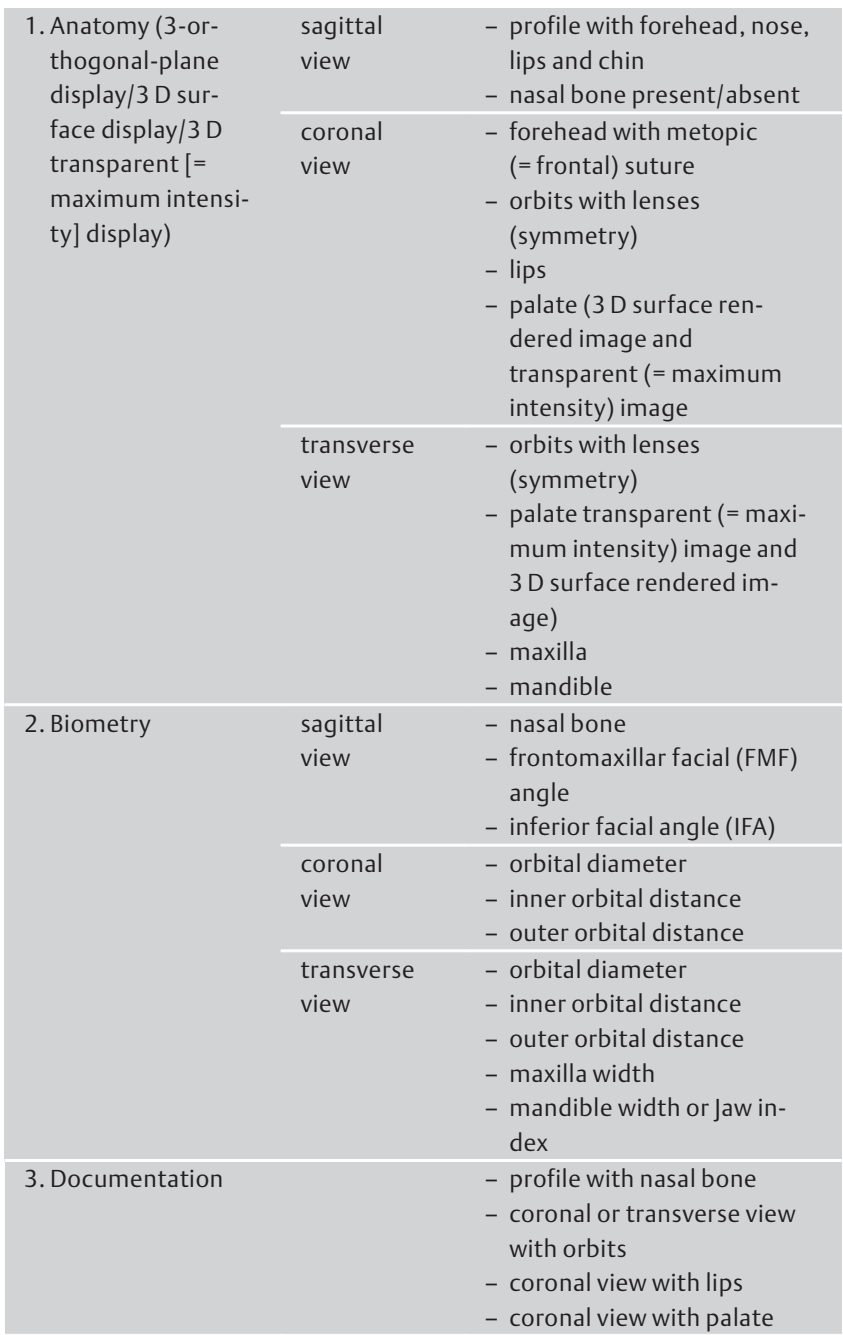

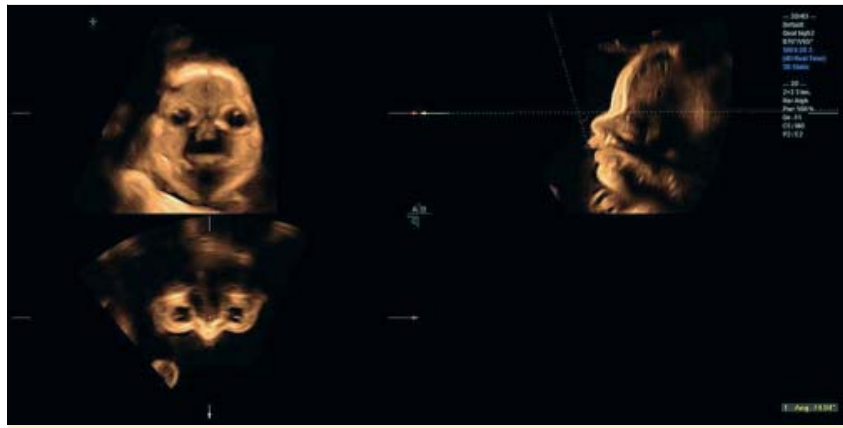

Fig. 17 Three-orthogonal-plane of a fetus at 29 weeks of gestation with normal inferior facial angle (IFA) of $74^{\circ}$. The IFA is defined on a sagittal view by the crossing of two lines: 1 . a line orthogonal to the vertical part of the forehead, drawn at the level of the synostosis of the nasal bones (reference line), 2. a line joining the tip of the mentum and the anterior border of the more protrusive lip (profile line). After Rotten et al. [28].

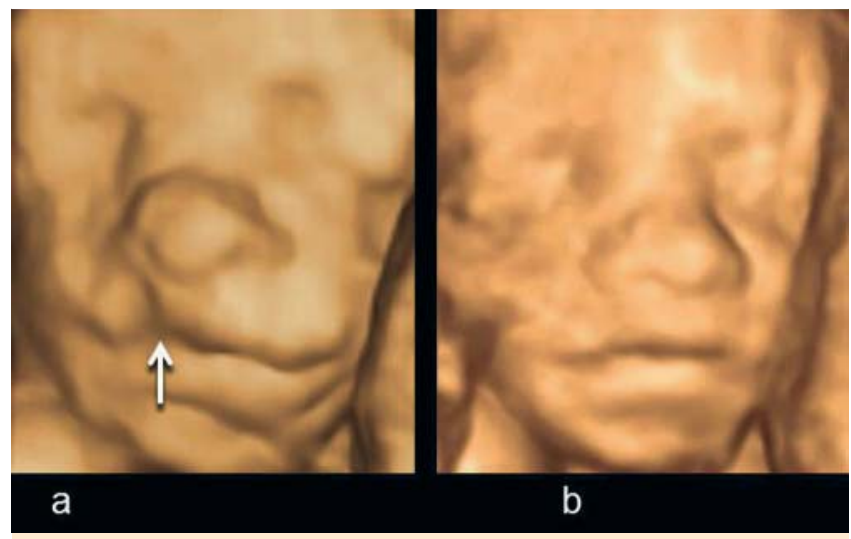

Fig. 18 a Movement artifact feigning a cleft lip right (white arrow). Surface-rendered frontal face view at 23 weeks of gestation. $\mathbf{b}$ The same fetal face a few seconds later in a quit position of the fetus, demonstrating normal lips.

differentiation between isolated cleft lip and cleft lip with cleft palate it is necessary to remove the frontal aspect of the fetal face electronically or to place the volume box inside the fetal face ( $\bullet$ Fig. 16]. This procedure enables an assessment of the fetal palate by providing an inner surface view, which enables the ready detection of a palatal defect. The diagnosis of cleft palate can also be established by different $3 \mathrm{D}$ US procedures as described by Campbell et al. [20], Benacerraf et al. [21], Platt et al. [22], Pilu et al. [23] and Ramos et al. [24]. The reverse face view [20] is also useful to detect anophthalmia [27].

For the sonographic recognition and characterization of fetal retrognathia and micrognathic mandibles in utero the use of the inferior facial angle (IFA) [28] ( $\bullet$ Fig. 17) and the mandible width/ maxilla width ratio [28] or the jaw index [29] can be used.

$4 \mathrm{D}$ US allows demonstration of a fetal defect in the moving fetus. After 4D US acquisition, not only one but several volumes are stored sequentially in a $4 \mathrm{D}$ volume cine loop. This $4 \mathrm{D}$ cine loop allows the examiner to repeatedly display the cine sequence at different speeds and to scroll back through several volumes to achieve the best $3 \mathrm{D}$ surface demonstration of the ROI [6]. Transparent (= maximum intensity) display enables the demonstration of normal and abnormal ossification of the fetal frontal

bones, abnormal width of the metopic (= frontal) suture, and absent or low ossification of nasal and other facial bones $[6,11,12]$.

\section{Limitations and pitfalls \\ $\nabla$}

Despite the fact that the different display modes enable an excellent demonstration of the fetal face there are a few situations that can cause problems [30]: 1. Fetal or probe movements during data acquisition lead to motion artifacts ( Fig. 18), 2. surface rendering cannot be done in severe oligohydramnios, 3. overlying or adjacent structures interfere with surface rendering and must first be removed with the electronic scalpel, 4 . arms or legs in front of the fetal face can cause shadowing artifacts defects because of the long bones, 5 . setting the threshold too high or faulty manipulation of the electronic scalpel leads to iatrogenic structural defects and 6 . the surface display of the fetal face in the early embryologic development (first trimester or beginning of the second trimester) does not show the typical human facial appearance as observable in the late second or in the third trimester and this should not be misinterpreted as a malformation of the fetal face. 


\section{Conclusions}

$3 \mathrm{D} / 4 \mathrm{D}$ ultrasound with its different display and volume manipulation possibilities offers a unique opportunity to demonstrate the normal and abnormal fetal face in detail. In cases with facial pathology this technique will help in appreciating the severity of the anomaly and in communicating of the defect with the neonatologist, the face surgeon and the parents. However, like in every imaging technology, it is necessary to know the limitations of $3 \mathrm{D} / 4 \mathrm{D}$ scanning and to be aware of possible pitfalls.

\section{References}

1 Pretorius DH, House M, Nelson TR et al. Evaluation of normal and abnormal lips in fetuses: Comparison between three- and two-dimensional sonography. Am J Roentgenol 1995; 165: 1233-1237

2 Merz E, Weber G, Bahlmann F et al. Application of transvaginal and abdominal three-dimensional ultrasound for the detection or exclusion of malformations of the fetal face. Ultrasound Obstet Gynecol 1997; 9: $237-243$

3 Rotten D, Levaillant JM. Two- and three-dimensional sonographic assessment of the fetal face. 1. A systematic analysis of the normal face. Ultrasound Obstet Gynecol 2004; 23: 224-231

4 Rotten D, Levaillant JM. Two-and three-dimensional sonographic assessment of the fetal face. 2. Analysis of cleft lip, alveolus and palate. Ultrasound Obstet Gynecol 2004; 24: 402 -411

5 Mittermayer C, Blaicher W, Brugger PC et al. Foetal facial clefts: Prenatal evaluation of lip and primary palate by 2D and 3D ultrasound. Ultraschall in Med 2004; 25: 120-125

6 Merz E, Welter C. 2D and 3D ultrasound evaluation of normal and abnormal fetal anomaly in the second and third trimesters in a level III center. Ultraschall in Med 2005; 26: 9-16

7 Merz E. Current 3D/4D ultrasound technology in prenatal diagnosis. Eur Clinics Obstet Gynaecol 2005; 1: 184-193

8 Merz E, Welter C, Oberstein A. 3D/4D Ultrasound Assessment of Fetal Face Malfor-mations. Medimond - Bologna, Italy: 2008, 29-37

9 Merz E, Benoit B, Blaas HG et al. Standardization of three-dimensional images in obstetrics and gynecology: consensus statement. Ultrasound Obstet Gynecol 2007; 29: 697-703

10 Merz E, Miric-Tesanic D, Welter C. Value of the electronic scalpel (cut mode) in the evaluation of the fetal face. Ultrasound Obstet Gynecol 2000; 16: $564-568$

11 Benoit B, Chaoui R. Three-dimensional ultrasound with maximal mode rendering: a novel technique for the diagnosis of bilateral or unilateral absence or hypoplasia of nasal bones in second-trimester screening for Down Syndrome. Ultrasound Obstet Gyneco1 2005; 25: 19-24

12 Faro C, Benoit B, Wegrzyn P et al. Three-dimensional sonographic description of the fetal frontal bones and metopic suture. Ultrasound $\mathrm{Ob}-$ stet Gynecol 2005; 26: 618-621
13 Achiron $R$, Gindes L, Zalel $Y$ et al. Three- and four-dimensional ultrasound: new methods for evaluating fetal thoracic anomalies. Ultrasound Obstet Gynecol 2008; 32: 36-43

14 Sonek JD, Nicolaides KH. Prenatal ultrasonographic diagnosis of nasal bone abnormalities in three fetuses with Down syndrome. Am J Obstet Gynecol 2002; 186: 139-141

15 Cicero S, Avgidou K, Rembouskos G et al. Nasal bone in first-trimester screening for trisomy 21. Am J Obstet Gynecol 2006; 195: 109-114

16 Sonek J, Borenstein M, Downing C et al. Frontomaxillary facial angles in screening for trisomy 21 at $14-23$ weeks' gestation. Am J Obstet Gynecol 2007; 197: 160.e1 - 160.e5

17 Borenstein $M$, Persico $N$, Kagan $K O$ et al. Frontomaxillary facial angle in screening for trisomy 21 at $11+0$ to $13+6$ weeks. Ultrasound Obstet Gynecol 2008; 32: 5-11

$18 \mathrm{Merz}$ E. The fetal nasal bone in the first trimester - precise assessment using 3D sonography. Ultraschall in Med 2005; 26: 365-366

19 Kagan KO, Cicero S, Staboulidou I et al. Fetal nasal bone in screening for trisomies 21, 18 and 13 and Turner syndrome at 11-13 weeks of gestation. Ultrasound Obstet Gynecol 2009; 33: 259-264

20 Campbell S, Lees C, Moscoso G et al. Ultrasound antenatal diagnosis of cleft palate by a new technique: the 3D "reverse face" view. Ultrasound Obstet Gynecol 2005; 25: $12-18$

21 Benacerraf BR, Sadow PM, Barnewolt CE et al. Cleft of the secondary palate without cleft lip diagnosed with three-dimensional ultrasound and magnetic resonance imaging in a fetus with Fryns' syndrome. Ultrasound Obstet Gynecol 2006; 27: 566 - 570

22 Platt $L D$, Devore GR, Pretorius DH et al. Improving cfleft palate/cleft lip antenatal diagnosis by 3-dimensional sonography: the "flipped face" view. J Ultrasound Med 2006; 25: 1423 - 1430

23 Pilu G, Segata M. A novel technique for visualization of the normal and cleft fetal secondary palate: angled insonation and three-dimensional ultrasound. Ultrasound Obstet Gynecol 2007; 29: 166-169

24 Ramos GA, Romine LE, Gindes L et al. Evaluation of the Fetal Secondary Palate by 3-Dimensional Ultrasonography. J Ultrasound Med 2010; 29: 357-364

25 Lee W, McNie B, Chaiworapongsa T et al. Three-dimensional ultrasonographic presentation of micrognathia. J Ultrasound Med 2002; 21: $775-781$

26 Dagklis T, Borenstein M, Peralta CF et al. Three-dimensional evaluation of mid-facial hypoplasia in fetuses with trisomy 21 at $11+0$ to $13+6$ weeks of gestation. Ultrasound Obstet Gynecol 2006; 28: 261 - 265

27 Wong HS, Parker S, Tait J et al. Antenatal diagnosis of anopthalmia by three-dimensionl ultrasound: a novel application of the reverse face view. Ultrasound Obstet Gynecol 2008; 32: 103 -105

28 Rotten D, Levaillant JM, Martinez $\mathrm{H}$ et al. The fetal mandible: a 2D and $3 \mathrm{D}$ sonographic approach to the diagnosis of retrognathia and micrognathia. Ultrasound Obstet Gynecol 2002; 19: 122 - 130

29 Paladini D, Morra T, Teodoro A et al. Objective diagnosis of micrognathia in the fetus: the jaw index. Obstet Gynecol 1999; 93: 382-386

30 Merz E. 3-D Ultrasound in Prenatal Diagnosis. In: Merz E (ed.) Ultrasound in Obstetrics and Gynecology. Vol.1: Obstetrics Stuttgart-New York: Thieme 2005, 515-528 